MATEC Web of Conferences 22,01016 (2015)

DOI: $10.1051 /$ matec conf/ 20152201016

(C) Owned by the authors, published by EDP Sciences, 2015

\title{
Method of Tower-less Calibration for Phase Parameter of Tracking Sys- tem
}

\author{
Tiezhi Ge \\ Space Academy of Harbin Industrial University, Harbin, Heilongjiang, China \\ Jiuquan Satellite Launch Centre, Jiuquan, Gansu, China
}

Mingshan Wei, Qiang Su, Longcheng Huang \& Gang Quan*

Jiuquan Satellite Launch Centre, Jiuquan, Gansu, China

\begin{abstract}
This paper introduced the principle of angle errors in tracking system. The demodulation was only related to signal envelop, and supported the phase calibration of radio star. And this paper which is combined with the commonly-used phase calibration methods, carried out the phase calibration of radio star and the satellite on the specific device, summarized the procedure of radio star for phase calibration and compared the accuracy of it. On the basis of analyzing the theory of phase calibration, the authors concluded the relationship between the parameter of phase calibration and the working frequency. Through utilizing the results of satellite phase calibration to verification and the results of phase calibration of radio star on full-band, and achieving the parameter of phase calibration without the calibration tower, it has a good engineering practice value.
\end{abstract}

Keywords: tracking system; phase calibration method; the radio star; wave path difference

\section{INTRODUCTION}

With the development of space technology, in order to adapt to the situation of deep space and high-orbit monitoring, the monitoring equipment is gradually developed to large antenna and multimode feed, and it presents a severe test for the phase calibration of the tracking system. There are three phase calibration methods: calibration tower, synchronous satellite and radio stars.

The calibration tower is typically used for small-diameter antenna. The principle of this method is that the beacon is placed in a calibration tower, antenna electrical axis alignsbeacon antenna, and to ensure signal output of Angle tracking receiver. The phase difference of it is measured and the phase difference is zero by adjusting the phase in a certain method. When the antenna aperture is large, the calibration tower can't satisfy the near-field conditions of the phase calibration. The cost is very large to build calibration tower which can meet the far-field calibration requirements of a large aperture antenna, and it is very difficult to achieve. So it is difficult to meet the testing requirements of large aperture antenna by calibration tower method.

In many practical cases, no appropriate calibration tower can be used to calibration, so "no tower calibration phase" is demanded. The frequency data of the known synchronous satellite are less, however, then phase calibration parameter of the multimode feed are varied with the signal frequency, it is difficult for practical use.

*Corresponding author: quangalax@163.com
The radio star method is to use the air radio star instead of the calibration tower beacon source to implement school phase. The radio stars have high orbit and wide range of radio frequency; they are an excellent source for the phase calibration. The noise of radio star is white noise, and spectrum can cover multiple frequency bands. However, due to higher requirements for equipment performance, the radio measuring equipment needs to be combined with their own performance to select suitable radio star for calibrating work. The solar noise is one of the commonly-used radio sources, and it can be detected by most radio measuring equipment.

In this article which points out an S-band tracking system which has large caliber and wide bandwidth, we propose a method of calculating the phase calibration values using wave path-difference. The latter two methods were tested. By comparing the results, the correspondence between the phase calibration parameter and the work frequency is revealed; the result shows that the radio star method can correct the phase errors of tracking system quickly and effectively.

\section{WORKING PRINCIPLE OF THE TRACKING SYSTEM}

\subsection{Working principle}

Simplified block diagram of tracking system is shown in Figure 1.

If the received signal is signal emits from the radio star, and the signal type is the same with the receiver

This is an Open Access article distributed under the terms of the Creative Commons Attribution License 4.0, which permits unrestricted use, distribution, and reproduction in any medium, provided the original work is properly cited. 


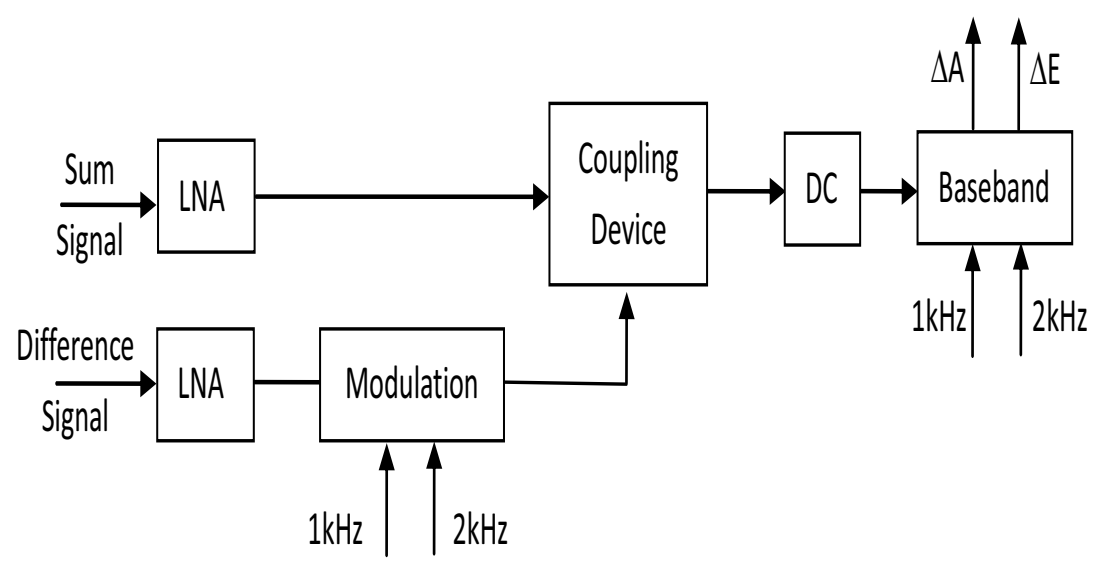

Figure 1. Simplified block diagram of tracking system

noise using the pseudo-random code approximate the statistical properties of the white noise, and then the signal of the radio star can be expressed as follows:

$S(t)=\sqrt{2 P} \mathrm{PN}(t) \cos \omega \mathrm{t}$

Where, $\omega$ is carrier angular frequency; $\mathrm{P}$ is channel power; PN is pseudo-random code. The complex expression of $\mathrm{S}(\mathrm{t})$ is shown as follows:

\section{$S(t)=A \exp [j(\omega t+\psi(t))]$}

When signal is amplified, modulated and coupling, the output pseudo pulse signal can be expressed as follows:

$$
\begin{aligned}
U_{c}= & a \sqrt{1-M} f_{s}(\theta) \exp [j(\omega t+\psi(t))] \\
& +A \sqrt{1-M} f_{d}(\theta) \exp [j(\omega t+\phi+\psi(t)-\pi / 2+\beta(t))]
\end{aligned}
$$

Where, $A$ is the amplitude of the sum signal; $\psi(t)$ is instantaneous phase shift of the angle adjusting signal; $M$ is coupling parameter; $f_{s}(\theta)$ is normalized sum pattern; $f_{d}(\theta)$ is normalized difference pattern; $\theta$ and $\phi$ are target's angular coordinate of spherical coordinates that the $\mathrm{Z}$ axes is the axis of the antenna. $\beta(t)$ is modulated signal. Gaussian white noise can be expressed as follows:

$$
n(t)=R(t) \cos [\omega t+\alpha(t)]=n_{c}(t) \cos \omega t-n_{c}(t) \sin \omega t
$$

Complex expression can be expressed as follows: $n(t)=R(t) \exp [j(\omega t+\alpha(t))]$

When $\theta$ is small, $f_{s}(\theta) \approx 1, f_{d}(\theta) \approx \mu \theta, \mu$ is slope of relative difference. Considering the Gaussian noise, the received signal changes are shown as follows:

$$
\begin{aligned}
U= & a \sqrt{1-M} f_{S}(\theta) \exp [j(\omega t+\psi(t))]+R(t) \exp [j(\omega t+\alpha(t))](6) \\
& +A \sqrt{M} f_{d}(\theta) \exp [j(\omega t+\phi+\psi(t)-\pi / 2+\beta(t))]
\end{aligned}
$$

So that the received signal can be expressed as $U=\mathrm{C}(t) \cos (\omega t+\varphi \mathrm{C}(t)), \mathrm{C}(t)$ is the signal envelope shown as follows:

$$
C(t)=\left\{\begin{array}{l}
A^{2}(1-M)+A^{2} M(\mu \theta)^{2}+R^{2}(t) \\
+2 A^{2} \sqrt{(1-M) M} \mu \theta \sin (\varphi+\beta(t)) \\
+2 A \sqrt{(1-M)} R(t) \cos [\psi(t)-\alpha(t)] \\
-2 A \sqrt{M} R(t) \mu \theta \sin [\alpha(t)-\varphi-\psi(t)-\beta(t)]
\end{array}\right\}^{1 / 2}
$$

Noise can be neglected if the signal-noise ratio is low:

$$
\begin{aligned}
C(t) & =R(t)+1 / 2 R(t)\left[A^{2}(1-M)+A^{2} M(\mu \theta)^{2}\right. \\
& \left.+2 A^{2} \sqrt{(1-M) M} \mu \theta \sin (\phi+\beta(t))\right] \\
& +A \sqrt{(1-M)} \cos [\psi(t)-\alpha(t)] \\
& -A \sqrt{M} \mu \theta \sin [\alpha(t)-\psi(t)-\beta(t)+\phi]
\end{aligned}
$$

When the receiver received the sun signal which is broadband noise, carrier can't be locked, and this situation was considered and resolved when the tracking system was designed. After loading parameters, the tracking system can correct the phase and output voltage errors without carrier locked. The format of multimode feed is non-coherent envelope demodulation, and this format which is unaffected by the signal form (frequency modulation, spread spectrum and broadband noise) is only related with the signal envelope, so it can correct phase when the received signal is a broadband noise.

\subsection{Self-tracking parameters}

Self-tracking parameters include phase and differential slope, the calibration of parameters is often referred to as "calibration phase". In high-precision angle measuring device of radio tracking systems, a single pulse amplitude system is often used. And inconsistencies of its phase difference between channels will lead to the following results: First, the directional sensitivity of angle error decreases; second, the angle measurement error is brought by cross-coupled one into a single channel monopulse angle tracking system.

The error voltage of cross-coupling is an important 
indicator of a tracking system. It directly affects the convergence time of target tracking antenna. If the error voltage is too large, the convergence time will be long, and the tracking error is increased with it. Finally, the goal would be not tracked. Hence in order to stable self-tracking of radio measurement equipment, calibration phase that the phase difference between the signals is zero is necessary.

According to engineering experience, the error voltage of cross-coupling is small, and the tracking performance of antenna is superior at $\tan \Phi<1 / 7(\Phi$ is the difference between the phase difference and the calibration phase parameters). When the error voltage of cross-coupling is relatively small, the tracking performance of antenna is relatively good at $\tan \Phi<1 / 5$. When the error voltage of cross-coupling is relatively large, the tracking performance of antenna is only available at $\tan \Phi<1 / 3$. When the error voltage of cross-coupling is too large, the antenna cannot be tracked at $\tan \Phi<1 / 3$. In the different values of $\Phi$, convergence curves of some tracking system are shown in Figure 2, Figure 3 and Figure 4.

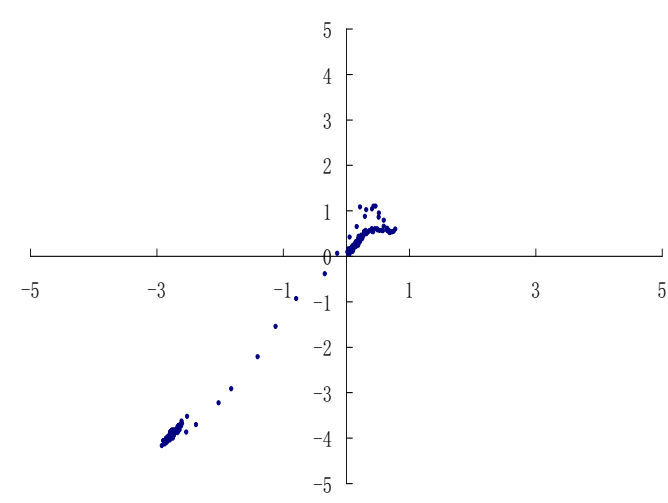

Figure 2. Convergence curves of tracking system at $\Phi=5^{\circ}$

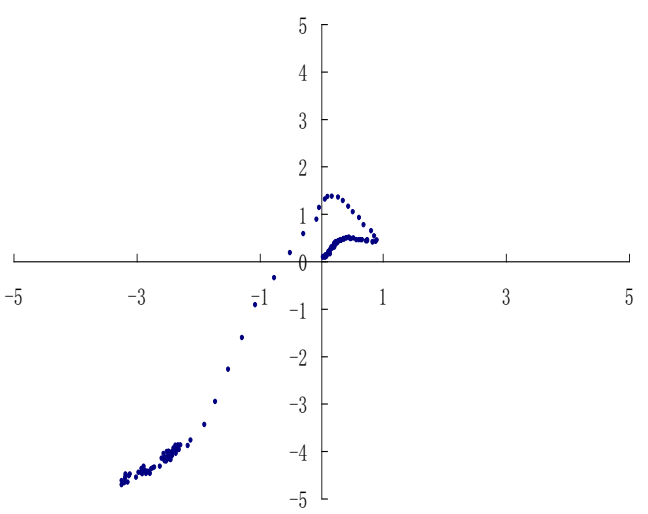

Figure 3. Convergence curves of tracking system at $\Phi=15^{\circ}$

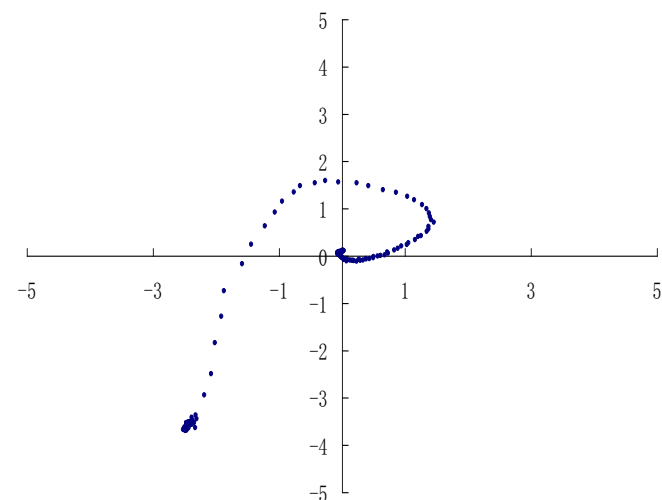

Figure 4. Convergence curves of tracking system at $\Phi=25^{\circ}$

\section{EXPERIMENTS OF PHASE CALIBRATION USING THE RADIO STARS}

\subsection{Radio star selection}

The radio star has some features: all-day, wide distribution and ephemeris which is precisely known, they are suitable for the phase calibration of the tracking system, but this method has certain requirements for the performance of the tracking system, it is shown in Table 1.

Table 1.Minimum G/T of the tracking system by using different radio star

\begin{tabular}{ll}
\hline Radio star & $\mathrm{G} / \mathrm{T}(\mathrm{dB} / \mathrm{K})$ \\
\hline Orion A & 39.1 \\
Cassiopeia A & 35.4 \\
Moon & 20 \\
Sun & 16 \\
\hline \multicolumn{2}{l}{ The phase calibration often uses the sun or the } \\
moon due to the low required value of $\mathrm{G} / \mathrm{T}$.
\end{tabular}

\subsection{Process of phase calibration using the radio star}

Based on the feasibility analysis of the solar calibration, the sun calibration phase model of radio measurement equipment is used for testing experimental. Solar calibration phase of radio measurement equipment is achieved, and the closed-loop self-tracking of radio measurement equipment is accomplished. Test equipment diagram is shown in Figure 5.

For example, pseudo single-pulse self-tracking system is used in angle error demodulation of some device baseband pseudo single-pulse self-tracking system; the synthesis method is a four-phase system; data signals is in recliner information of single channel signal; angle error tracking information is in amplitude information. The reference signals of $1 \mathrm{kHz}$ and $2 \mathrm{kHz}$ are used for synchronizing synchronization delay caused by angle modulation and channel transmission. Therefore, the local synchronous demodulation refer- 
ence signal and the input angle error modulation envelope are strict synchronizations. This will obtain high accuracy angle error signal AM.

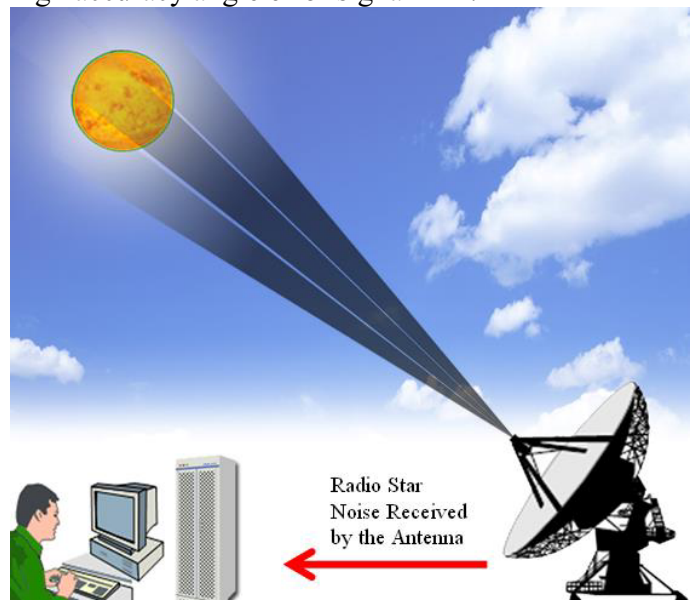

Figure 5.Equipment connection diagram of phase calibration by radio star

According to the correspondence of $1 \mathrm{kHz}$ and 2 $\mathrm{kHz}$, and ignoring the $\mathrm{DC}$ component, the relationship is obtained as follows:

$C_{1}=A \sqrt{M} u \theta \sin \phi=A \sqrt{M} u \Delta A$, at $1 \mathrm{kHz}=1,2 \mathrm{kHz}=1$

$C_{2}=A \sqrt{M} u \theta \cos \phi=A \sqrt{M} u \Delta E$, at $1 \mathrm{kHz}=1,2 \mathrm{kHz}=0$

$C_{3}=-A \sqrt{M} u \theta \sin \phi=-A \sqrt{M} u \Delta A$, at $1 \mathrm{kHz}=0,2 \mathrm{kHz}=1$

$C_{4}=-A \sqrt{M} u \theta \cos \phi=-A \sqrt{M} u \Delta E$, at $1 \mathrm{kHz}=0,2 \mathrm{kHz}=0$

The process of phase calibration using the radio star is shown in the following Figure 6:

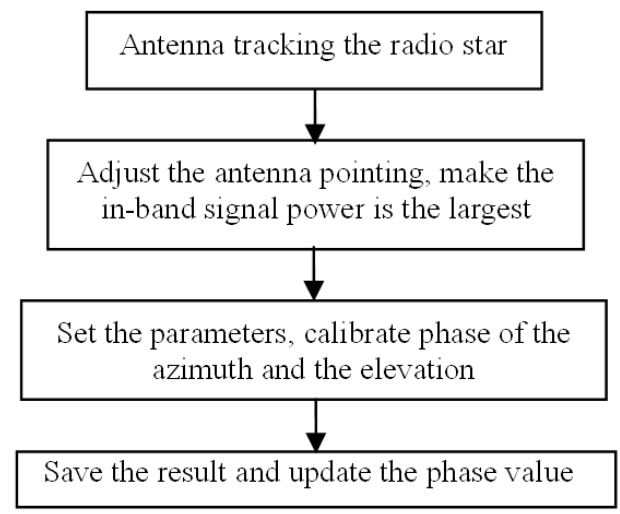

Figure 6.Process of phase calibration

It is important to note that:

(1)During the process of phase calibration, in order to make sure the zero is consistent, the antenna needs tracking the radio star in real time.

(2)In order to find the accurate zero of phase calibration, the plus and the minus shift $5 \mathrm{mil}$ at the strongest point of the noise power which are within the signal bandwidth.
(3)When the radio star elevation angle is too high, the azimuth difference slope needs secant compensation.

(4)Make more phase calibration experiments and take the mean.

\subsection{Phase calibration result analysis}

As a certain type of S-band tracking system correct phase using the sun, the results of frequency 220X.XMHz are enumerated in Table 2:

Table 2. Results of correct phase using the sun at frequency 220X.XMHz

\begin{tabular}{lll}
\hline Rotatio & Azimuth phase $\left(^{\circ}\right)$ & Elevation phase $\left(^{\circ}\right)$ \\
\hline Left & 338.38 & 167.17 \\
Right & 261.39 & 248.73 \\
Left & 352.62 & 147.83 \\
Right & 241.00 & 255.76 \\
Left & 357.36 & 145.72 \\
Right & 241.17 & 261.39 \\
Left & 336.09 & 165.94 \\
Right & 261.91 & 245.74 \\
\hline
\end{tabular}

The errors of single phase calibration using the sun are normally distributed, the cross-coupling index is 0.2 that means the conversion angle is $11.31^{\circ}$. Referred to Table 2, the probability that the result of single phase calibration falls within the available range which is shown in Table 3:

Table 3. Probability of the result that single phase calibration fall within the range of available

\begin{tabular}{lllll}
\hline Rotation & $\operatorname{Mean}\left({ }^{\circ}\right)$ & Variance $\left(^{\circ}\right)$ & $\mathrm{x}$ & $\varphi(\mathrm{x})$ \\
\hline Left Azimuth & 346.11 & 10.47 & 1.08 & 0.86 \\
Left Elevation & 156.67 & 11.46 & 0.99 & 0.84 \\
Right Azimuth & 251.36 & 11.87 & 0.95 & 0.83 \\
Right Elevation & 252.91 & 7.04 & 1.60 & 0.95 \\
\hline
\end{tabular}

As it can be seen from Table 3, the probability is between $83 \% \sim 95 \%$. It can't guarantee the result of single phase calibration to meet the cross-coupling index of 0.2 .

Experiments are made at the same frequency by using the satellite. The signal in rotation is right, and the result is $\left(255.23^{\circ}, 256.76^{\circ}\right)$. Compared with the result of phase calibration using the sun which are $\left(251.36^{\circ}\right.$, $\left.252.91^{\circ}\right)$, the difference are $\left(3.87^{\circ}, 3.85^{\circ}\right)$ which is less than $\pm 5^{\circ}$. After the calibration using the sun, the tracking system can track the satellite reliably, and meet the cross-coupling index of 0.2.

\section{CALCULATION METHOD OF THE PHASE CALIBRATION PARAMETER}

\subsection{Phase calibration analysis and calculation theory}

After modulation, the difference signal coupled the sum signal, so that the system can correct phase without carrier locked. By modulating, the sum signal and the difference signal can phase-coherent. However, 
after entering the feed, the sum signal and the difference signal go through different physical paths, and there is a fixed wave path difference which is the difference phase of the sum signal and the difference signal. So we can obtain the following formula for an arbitrary frequency $f_{1}$ :

$$
S=\lambda_{1} N_{1}+K_{1}
$$

Where, $S$ is the fixed wave path difference, $\lambda_{1}=\mathrm{c} / f_{1}$, $\lambda_{1} N_{1}$ is the integer times wavelength of phase; $K_{1}$ is allowance, $K_{1}=\lambda_{1} \theta_{1} / 360^{\circ}, \theta_{1}$ is the phase shift value which is the value of phase calibration.

At the frequency $f_{2}$, the fixed wave path difference can be acquired as follows:

$$
S=\lambda_{2} N_{2}+K_{2}
$$

So it is shown as follows:

$\lambda_{1} N_{1}+K_{1}=\lambda_{2} N_{2}+K_{2}$

That is shown as follows:

$\frac{c}{f_{1}} N_{1}+\frac{c}{f_{1}} \frac{\theta_{1}}{360}=\frac{c}{f_{2}} N_{2}+\frac{c}{f_{2}} \frac{\theta_{2}}{360}$

\subsection{Calculation method of the phase calibration pa- rameter}

As a certain type of tracking system correct phase using the satellite, the results of frequency 22XX.3 and $22 X X .7$ are $[(139.69,330.64),(69.02,48.93)]$ and $[(250.17,81.24),(180.28,161.40)]$, instead of $\theta_{1}$ and $\theta_{2}$ which are in the formula (10) and (11), then we can obtain the relationship between the fixed wave path difference $S$ and the integer N, and the relationship between $\mathrm{N}_{1}$ and $\mathrm{N}_{2}$ is shown in the following figures.

It can be seen in the Figure 3 that linear $\mathrm{N}_{1}$ and $\mathrm{N}_{2}$ are increased with the increase of $S$, and $\mathrm{N}_{1}$ and $\mathrm{N}_{2}$ are roughly equal. Make $\mathrm{N}_{1}=\mathrm{N}_{2}=\mathrm{N}$, so it is shown as follows:

$$
N=\left(\lambda_{2} \theta_{2}-\lambda_{1} \theta_{1}\right) / 360\left(\lambda_{1}-\lambda_{2}\right)
$$

After the calculation formula (13), $\mathrm{N}_{1}=\mathrm{N}_{2}=37$, $S=5.02 \mathrm{~m}$. When $S$ is determined, the phase calibration values of other frequency can be calculated by taking advantage of the $\mathrm{N}$ value as shown in the figure as follows (Azimuth of left-handed orientation):

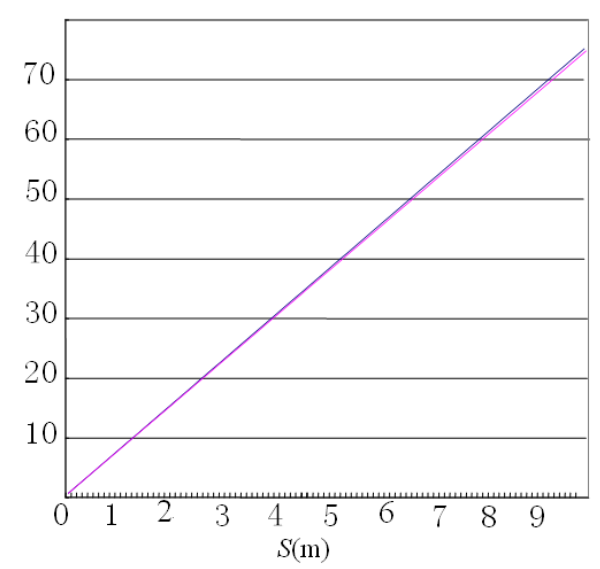

Figure 7. Changing trend of $\mathrm{N}$

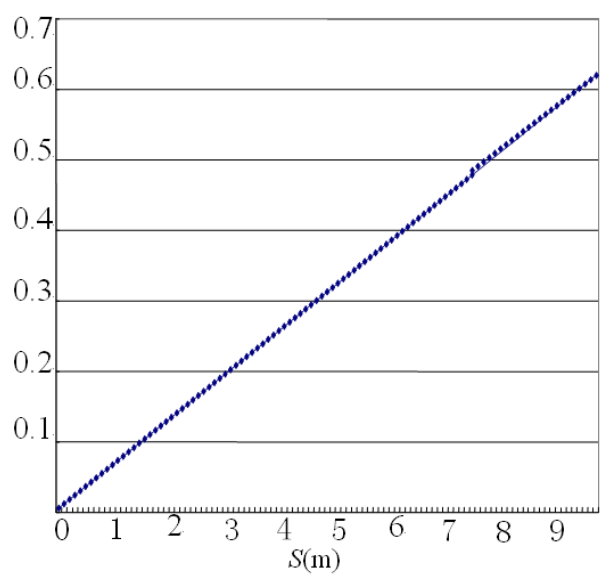

Figure 8. Difference of $\mathrm{N}_{1} \mathrm{andN}_{2}$

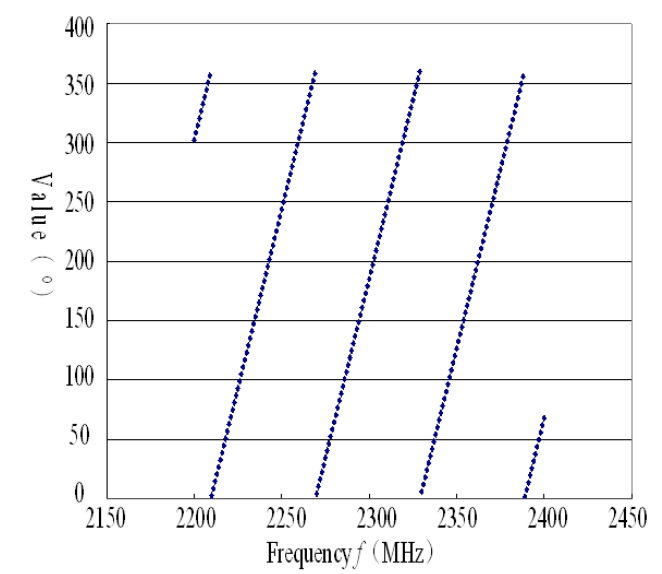

Figure 9. Phase calibration values of Azimuth of left-handed orientation

It can be seen from Figure 5 that the phase calibration results present linear change of $0^{\circ} \sim 360^{\circ}$ in a certain frequency cycle. It can be described as following formula:

$\theta_{A L}=6.0282 f+k \times 360$

Where, $\mathrm{k} \in \mathrm{Z}, \mathrm{k} \times 360$ ensure $\theta_{\mathrm{AL}} \in[0,360)$. In the same way, the formulas of other orientations are shown as follows:

$\theta_{E L}=5.9544 f+k \times 360(k \in Z)$

$\theta_{A R}=5.9976 f+k \times 360(k \in Z)$

$\theta_{E R}=5.9889 f+k \times 360(k \in Z)$

List facts by using either the style tag List signs or the style tag List numbers. 


\subsection{Results analysis}

Compared with the results of phase calibration using the sun, the phase calibration values of the tracking system can be calculated at some certain frequency by using the formulas (14 17). They are shown in Table 4:

Table 4. Comparison of the calculated results and the results of phase calibration using the sun

\begin{tabular}{llll}
\hline & Frequency(MHz) & $\begin{array}{l}\text { Calculate } \\
\text { values }\end{array}$ & $\begin{array}{l}\text { Results of } \\
\text { using the sun }\end{array}$ \\
\hline$\theta_{\mathrm{AR}}$ & $22 \mathrm{XX.3}$ & $274.7^{\circ}$ & $260.2^{\circ}$ \\
$\theta_{\mathrm{ER}}$ & $22 \mathrm{XX.3}$ & $254.5^{\circ}$ & $255.41^{\circ}$ \\
$\theta_{\mathrm{AR}}$ & $22 \mathrm{XX} .7$ & $99.63^{\circ}$ & $94.57^{\circ}$ \\
$\theta_{\mathrm{ER}}$ & $22 \mathrm{XX.7}$ & $80.16^{\circ}$ & $90.87^{\circ}$ \\
\hline
\end{tabular}

By comparison, the results of phase calibration use the sun and calculate values difference within $\pm 15^{\circ}$. After many tests and trials, the tracking system has accumulated large amounts of data of phase calibration using the sun, and the calculated results accord with the actual data. Through correcting the system phase using the calculated results, the tracking system can be stable to track the target such as the space station and synchronous satellite, and the angle error convergence is normal.

\section{PHOTOGRAPHS AND FIGURES}

By studying a certain type of S-band tracking system and the phase calibration using the radio star, this article pointed out an S-band tracking system which has large caliber and wide bandwidth, proposed a method of calculating the value of phase calibration using the sun by using the wave path-difference. Compared the calculated results with the actual phase calibration results, the trends are same and the errors are small. By tracking targets that validate the results, the results are available. This method of phase calibration using the sun can correct the phase errors of tracking system quickly and effectively.

\section{REFERENCES}

[1] Ding Lu-fei. \& Gen Fu-lu. 2003. Principle of radar, Publish house of Xi'an University of Electronic Science and Technology, Xi'an.

[2] Mao Nai-hong. 1987. Antenna measurement manual, Publish house of National Defense Industry.

[3] Dong Jian-wei, Gao Chang-sheng, Xiong Wei. 2013. Tower-less Calibration System Design of Pulse Radar, Computer Measurement and Control.

[4] Mao Nan-ping, Xu Chang-qin. \& Zhang Zhong-hua. 2004. Tower-less Calibration Technology for Vessel Track and Control Radar at Sea, Telecommunication Engineering.

[5] Hu Jin-dong. 2008. Research on phase-calibrating of tracking Radar, Journal of CAEIT, 6
[6] Liu Jia-xing. 2010. Tower-less Phase Calibration Using Radio Star Noise, Telecommunication Engineering, Vol.50, No.6. 